\section{Fibroblast Growth Factor 23}

H.-D. Haubeck

Wasserburg, Deutschland

Synonym(e) Fibroblasten-Wachstumsfaktor-23; FGF-23

Englischer Begriff fibroblast growth factor 23

Definition Fibroblast Growth Factor 23 (FGF-23), ein Mitglied der FGF-Genfamilie, spielt eine wichtige Rolle bei der Regulation des $>$ Phosphat-Stoffwechsels.

Beschreibung Die Serumkonzentrationen von $>$ Calcium und $>$ Phosphat werden in einem engen Konzentrationsbereich konstant gehalten. Während die Regulation der Calcium-Konzentration über Parathormon und 1,25 $[\mathrm{OH}]_{2}$-Vitamin D ( $\triangleright$ Vitamin D) bereits seit Langem bekannt ist, zeichnet sich erst jetzt ein Verständnis der Mechanismen der Phosphatregulation ab. Die Bedeutung von Fibroblast Growth Factor 23 (FGF-23) für die Phosphatregulation ergab sich aus Untersuchungen bei Patienten mit X-chromosomal vererbter Osteomalazie (Rachitis) (XLH), autosomal dominant vererbter hypophosphatämischer Osteomalazie (Rachitis) (ADHR) und tumorinduzierter Osteomalazie (TIO). Diesen Krankheitsbildern gemeinsam sind eine Hypophosphatämie als Folge einer erhöhten Phosphatausscheidung in den Urin und Mineralisationsstörungen der Knochen. Die bei diesen Patienten in der Regel normalen Serumkonzentrationen von Calcium und Parathormon, aber auch die Normalisierung der Befunde bei Patienten mit TIO nach Entfernung des Tumors wiesen auf einen phosphaturischen Faktor (Phosphatonin) hin. FGF-23 wurde als verantwortlicher Faktor aus Tumoren von Patienten mit TIO identifiziert. Durch FGF-23 lassen sich eine erhöhte Phosphatausscheidung in den Urin und eine Hypophosphatämie auslösen. Außerdem wird über die Inhibition der 1- $\alpha$-Hydroxylase der Niere durch FGF-23 auch die 1,25[OH $]_{2}$-Vitamin-D-Serumkonzentration beeinflusst. Tierexperimentell lassen sich durch FGF-23 die Symptome der genannten Krankheiten auslösen. Darüber hinaus werden nicht nur bei Patienten mit TIO, sondern auch bei Patienten mit XLH erhöhte Konzentrationen von FGF-23 nachgewiesen. Bei Patienten mit ADHR führen Mutationen im FGF-23-Gen dazu, dass FGF-23 nicht mehr proteolytisch abgebaut werden kann. Mit ,secreted frizzled-related protein 4" wurde aus Tumoren von Patienten mit TIO ein weiteres Phosphatonin identifiziert, das ebenfalls eine Phosphaturie und eine Hypophosphatämie verursacht.

Für die Messung der FGF-23-Konzentration im Serum steht ein Enzymimmunoassay zur Verfügung.

\section{Literatur}

Berndt T, Craig TA, Bowe AE et al (2003) Secreted frizzled-related protein 4 is a potent tumor-derived phosphaturic agent. J Clin Invest 112:785-794

Shimada T, Mizutani S, Muto Tet al (2001) Cloning and characterization of FGF23 as a causative factor of tumor-induced osteomalacia. Proc Natl Acad Sci U S A 98:6500-6505

Shimada T, Kakitani M, Yamazaki Y et al (2004) Targeted ablation of FGF-23 demonstrates an essential physiological role of FGF-23 in phosphate and vitamin D metabolism. J Clin Invest 113:561-568 